\title{
Fibre segment interferometry using code-division multiplexed optical signal processing for strain sensing applications
}

\author{
Thomas Kissinger, Thomas O. H. Charrett and Ralph P. Tatam \\ Department of Engineering Photonics, School of Engineering \\ Cranfield University, Cranfield, MK43 0AL, United Kingdom
}

\begin{abstract}
A novel optical signal processing scheme for multiplexing fibre segment interferometers is proposed. The continuous-wave, homodyne technique combines code-division multiplexing with single-sideband modulation. It uses only one electro-optic phase modulator to achieve both range separation and quadrature interferometric phase measurement. This scheme is applied to fibre segment interferometry, where a number of long-gauge length interferometric fibre sensors are formed by subtracting pairs of signals from equidistantly placed, weak back reflectors. In this work we give a detailed account of the signal processing involved and, in particular, explore aspects such as electronic bandwidth requirements, noise, crosstalk and linearity, which are important design considerations. A signal bandwidth of $\pm 20 \mathrm{kHz}$ permits the resolution of phase change rates of 2.5 . $10^{4} \mathrm{rad} \mathrm{s}^{-1}$ for each of the four $16.5 \mathrm{~m}$ long segments in our setup. We show that dynamic strain resolutions below 0.2 nanostrain $\cdot(\mathrm{Hz})^{-0.5}$ at $2 \mathrm{~m}$ sensor gauge length are achievable, even with an inexpensive diode laser. When used in applications that require only relative strain change measurements, this scheme compares well to more established techniques and can provide high-fidelity yet cost-effective measurements.
\end{abstract}

\section{Keywords:}

Interferometric signal processing, single-sideband signal processing, code-division multiplexing, fibre segment interferometry, dynamic strain sensing, long-gauge length fibre strain sensor

\section{Introduction}

In fibre segment interferometry (FSI), consecutive segments of fibre are separated by weakly back reflecting nodes that act as interferometric signal sources. The phase signals from two neighbouring reflectors can be subtracted, resulting in a measurement of the optical path difference (OPD) integrated over the whole length of the fibre segment between the reflectors. The fibre segments can thus be thought of as long-gauge length fibre sensors and any physical effect that changes the OPD such as strain and temperature can be measured. Provided that reliable in-fibre reflectors can be integrated into the sensing fibre without much effort, this type of sensor configuration has the potential to be very economical. This approach has been used before in optical fibre hydrophone applications, where time-division multiplexing (TDM) [1] or a combination of TDM and wavelength division multiplexing (WDM) [2] were employed to separate the signals from different reflecting nodes.

However, FSI also has great potential for application in fibre optic strain measurement [3], an area 
currently dominated by localized sensors like fibre Bragg gratings (FBG) [4] or distributed techniques based on Rayleigh and Brillouin scattering [5, 6]. Our paper provides a complementary approach aiming to bridge the gap between fully localized and fully distributed techniques with a cost-effective solution for quantitative and high-speed measurements of the OPD change of long-gauge length fibre sensors. As we will show, the high intrinsic resolution of interferometric long-gauge length strain sensors allows for strain measurements that can compete well with non-interferometric FBG sensors in terms of dynamic strain sensitivity. This can be achieved even when using an inexpensive diode laser that has laser frequency noise levels orders of magnitudes higher [7] than those lasers used in high-end interferometric optical fibre hydrophone applications. Furthermore, it has been recognized that long-gauge length sensors can have advantages over localized sensors when, for example, the presence of cracks should be detected [8]. However, unlike FBGs, but common to all single-wavelength interferometric phase measurement techniques, there is no absolute reference point. In practice, this restricts the proposed approach to the measurement of relative strain changes. Potential applications for relative strain change measurements could be vibration based condition monitoring [9], dynamic strain sensing for actuator control in smart wind turbines blades [10] or for active vibration control of civil engineering structures [11]. Also, the technique presented in this work could be combined with FBG sensors operating at other wavelengths to obtain both short-gauge and long-gauge length strain information from the same sensing fibre.

Code-division multiplexing (CDM) is a group of multiplexing techniques that are widely used in communications systems, where they are known as code-division multiple access (CDMA), allowing several senders to transmit data simultaneously over one channel using modulation with a pseudo-random code. CDMA has gained widespread use in areas such as wireless communications and global positioning systems [12], however, in optical signal processing for metrology, there have only been a few reported applications of CDM techniques [13-20]. CDM techniques for interferometric sensors can be grouped into intensitymodulated [14-16] and phase-modulated schemes [17-20]. Phase-modulated schemes allow full quadrature interferometric phase measurement, which is required for the applications we propose, while intensity-based systems can only measure the real part of the interferometric phase.

In the novel scheme proposed here we combine phase-modulated CDM with single-sideband (SSB) homodyne signal processing $[21,22]$. SSB is a generic theory for determining the shape and scale of a periodic phase modulation waveform so that it can be used to emulate a heterodyne carrier over a bandlimited frequency region. Unlike the widely used phase generated carrier method [23] for signal processing, only one carrier frequency is used in SSB, which make it easier to adapt to CDM. By using CDM as a multiplexing technique, the use of costly pulsed or wavelength-tuneable lasers can be avoided by employing a simple continuous-wave, single-frequency laser diode. Also, by combining CDM with SSB homodyne signal processing, both multiplexing and signal processing can be performed using only a single standard phase modulator, thus eliminating the need for an additional Bragg cell [17] or having to use a more complicated quadrature phase modulator [18] as in other phase-modulated CDM techniques.

In section 2 we outline the theory of combining SSB with CDM. A particular focus is given to the requirements that are imposed by the use of anti-aliasing filtering necessary in digital signal processing. We show that the effects of filtering on code separation can be simply analysed using the impulse response of the anti-aliasing filter and that its influence can be mitigated by the introduction of guard times at the code symbol transitions. In section 3 the experimental setup and signal processing are described in detail, while in section 4 experimental results of quantitative noise, crosstalk and dynamic response measurements are presented. Also a strain measurement that is expected to be exemplary of the applications we envisage for this scheme is shown. Finally, key signal processing parameters, which, in future implementations, could be improved to achieve a higher spatial resolution or higher signal bandwidth, are discussed before concluding the paper in section 5 . 


\section{Theory}

\subsection{SSB signal processing}

SSB homodyne signal processing [21, 22] is a theory describing the shape and scale that a periodic, phase-modulated waveform must possess to emulate a heterodyne carrier of frequency $f$. This emulation can be achieved when the phase modulation waveform of suitable shape is scaled until one of the two carrier sidebands, either at positive $+f$ or negative frequency $-f$, disappears. It is then said to be in linear operation and no cyclic error occurs. The well-known pseudo-heterodyne technique $[24,25]$ using serrodyne modulation can also be described using the SSB equations. In general, when a periodic phase modulation is applied, there will be a comb of carrier frequencies at harmonics of the repetition frequency $f_{\mathrm{wf}}$ of the phase modulation waveform. Thus, equivalence to a heterodyne carrier can only be given for interferometric signals in a limited frequency region between $\pm 0.5 f_{\mathrm{wf}}$. Care has to be taken that no interferometric signals at higher frequencies occur because these can be folded back into the signal band by the other carriers of the comb. In principle, any harmonic of the carrier waveform can be chosen for the demodulation of the phase signal if the linearity conditions can be met. Following the derivation in [21,22], the detected interferometric signal $e_{n}(t)$, expanded into a complex Fourier series and band pass filtered around the chosen carrier harmonics $\pm n f_{\mathrm{wf}}$ with filter bandwidth $\pm 0.5 \cdot f_{\mathrm{wf}}$, can be written in the form:

$$
e_{n}(t) \propto R e\left\{c_{+n} \exp \left[\mathrm{i}\left(2 \pi n f_{\mathrm{wf}} t+\theta\right)\right]+c_{-n} \exp \left[\mathrm{i}\left(-2 \pi n f_{\mathrm{wf}} t+\theta\right)\right]\right\}
$$

Here, $n$ is the index of the chosen carrier harmonic in regard to the waveform repetition frequency $f_{\mathrm{wf}}$ and $\theta$ is the interferometric phase to be measured. The complex coefficients $c_{ \pm n}$ are given by:

$$
c_{n}=\frac{1}{2 \pi} \int_{-\frac{T_{\mathrm{wf}}}{2}}^{\frac{T_{\mathrm{wf}}}{2}} g(t) \exp \left[\mathrm{i}\left(s \cdot \varphi_{\mathrm{wf}}(t)-2 \pi n f_{\mathrm{wf}} t\right)\right] d t
$$

$g(t)$ is an optional real amplitude modulation function, $s$ and $\varphi_{\mathrm{wf}}(t)$ are the phase scaling factor and normalized modulation waveform respectively. Both $g(t)$ and $\varphi_{\mathrm{wf}}(t)$ are periodic with period $T_{\mathrm{wf}}=f_{\mathrm{wf}}{ }^{-1}$. The scaling factor $s$ will be tuned until one of the coefficients $c_{ \pm n}$ vanishes and thus linear operation is achieved. This is always possible when certain symmetry conditions on $\varphi_{\mathrm{wf}}(t)$ and $g(t)$ are met [21].

\subsection{CDM and m-sequence pseudo-random codes}

In code division multiplexing (CDM) the outgoing signal is encoded with a digital code, and the signal returning from a particular range is recovered using a time delayed code version, the decoding function $d(t-\tau)$ with a time delay $\tau$ equal to the time-of-flight of the signal. In this and in many other works on CDM for optical sensing applications, a special class of pseudo-random sequence, known as maximal length sequences or $\mathrm{m}$-sequences [26] is used for the digital code. M-sequences exist only for lengths $m=\left(2^{p}-1\right)$, where $p$ is any positive integer. In CDM the autocorrelation function can be used to mathematically express the expected code overlap between the code at encoding and a delayed version at decoding. The special autocorrelation properties of $\mathrm{m}$-sequences result in a constant autocorrelation value for any non-zero code delay. The amplitude suppression ratio $\mathrm{R}$ is the ratio between autocorrelation values at zero and non-zero code delays, i.e. between in-range and out-of-range signals. A bipolar $(-1 ; 1) \mathrm{m}$-sequence is derived from the original unipolar $(0 ; 1) \mathrm{m}$-sequence by exchanging every 0 with a -1 . It was first described in Kersey et al. [15] that decoding a signal with a unipolar $\mathrm{m}$-sequence that was encoded with a bipolar $\mathrm{m}$-sequence or vice versa will have an infinitely low amplitude suppression ratio resulting in theoretically zero crosstalk between 
range channels. This shall be referred to here as unipolar-bipolar coding with amplitude suppression ratio $R_{\mathrm{ub}}$. In contrast, other CDM schemes often use bipolar-bipolar coding with suppression ratio $R_{\mathrm{bb}}$. Both $R_{\mathrm{ub}}$ and $R_{\mathrm{bb}}$ in $\mathrm{dBs}$ for a m-sequence of length $m$ are given by [15]:

$$
\begin{aligned}
& R_{\mathrm{bb}}=-20 \log _{10}(m) \\
& R_{\mathrm{ub}}=-\infty
\end{aligned}
$$

\subsection{Extended SSB equations for combining SSB and CDM}

In this work, we combine SSB with phase-modulated CDM and incorporate the effects of anti-aliasing filtering into the theory. This requires the SSB equations (1) and (2) to be extended. In the interferometric setup used in this work the non-stationary signal $e_{\mathrm{pd}, i}(t)$ at the photo detector that originates from the interference between the local oscillator (LO) and the back reflection from source $i$ can be written as:

$$
e_{\mathrm{pd}, i}(t)=A_{i} \cdot \operatorname{Re}\left\{g\left(\mathrm{t}-t_{i}\right) \cdot \exp \left[\mathrm{i}\left(s \cdot \varphi_{\mathrm{c}}\left(t-t_{i}\right)+\theta_{i}\right)\right]\right\}
$$

Here, $A_{i}=2 \sqrt{P_{\mathrm{LO}} P_{i}} \cdot R \cdot V_{i}$ is the interferometric signal amplitude for the signal originating from source $i$, which depends on the LO power $P_{\mathrm{LO}}$, reflected light power $P_{i}$, responsivity $R$ of the photo detector and the visibility $V_{i} . V_{i}$ is related to mutual polarization overlap and coherence properties between the source $i$ and the LO. $g\left(\mathrm{t}-t_{i}\right)$ is an optional periodic intensity modulation function. $s$ is the phase modulation scaling factor and $\varphi_{c}\left(t-t_{i}\right)$ is the normalized phase modulation waveform, delayed by the time-of-flight $t_{i}$ to source $i$ and back. Finally, $\theta_{i}$ is the interferometric phase signal of source $i$ that is to be measured.

Note that, in order to incorporate CDM coding, the phase modulation waveform $\varphi_{\mathrm{c}}(t)$ now contains many individual SSB waveforms that can be grouped into $m$ code symbols of length $T_{\text {symb }}$, each containing $k$ individual SSB waveforms $\varphi_{\mathrm{wf}}(t)$ of length $T_{\mathrm{wf}}$. Note that $\varphi_{\mathrm{c}}(t)$ is now periodic with the repetition frequency $f_{\mathrm{c}}=T_{\mathrm{c}}^{-1}$ of the whole code. Figure 1) shows an illustration of typical signals at each processing step for the signal originating from one source $i$. The applied phase modulation is shown in figure 1a) and the resulting signal recorded at the detector is shown in $1 \mathrm{~b}$ ) for an interferometer's cosine-shaped transfer function and an arbitrary phase angle of $\theta_{i}=60^{\circ}$. In the following derivation the phase signal $\theta_{i}$ is assumed to be stationary, an assumption also used in $[21,22]$ that will be further discussed at the end of this section. 

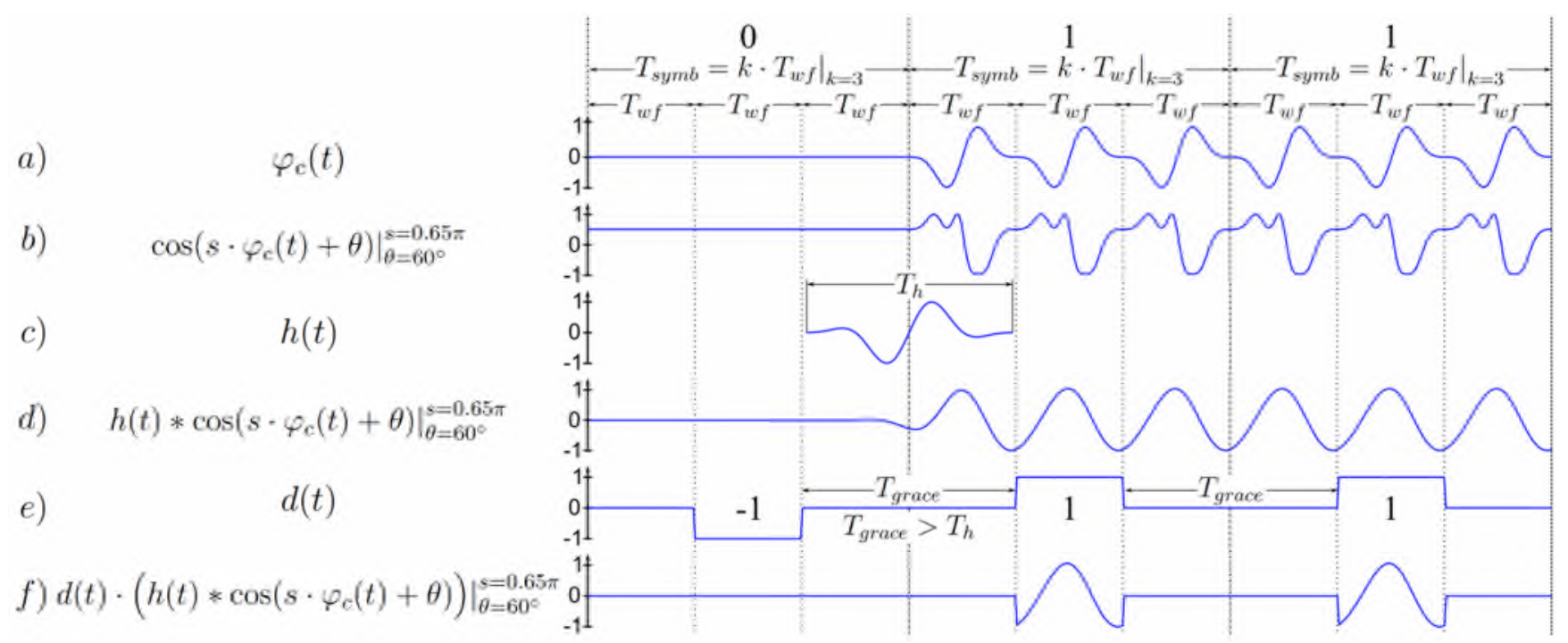

Figure 1. Illustration of typical signal shapes at the intermediate stages of signal processing for the combined SSD$\mathrm{CDM}$ waveform $\varphi_{\mathrm{c}}(t)$ used in the later experiments. All signals are normalized and show how a sequence of unipolar code $0,1,1$, an excerpt of a longer code of length $T_{\mathrm{c}}$, is modulated, photo detected, filtered and finally decoded using the bipolar code $-1,1,1$.

After detection the electronic signal is subjected to a filter with an impulse response $h(t)$, figure 1c), that is zero for any point in time outside the filter length $T_{h}$. Anti-aliasing filtering is necessary for the conversion from continuous-time to discrete-time signals to satisfy the Nyquist-Shannon sampling theorem [18] and also to limit the noise bandwidth. The filter $h(t)$ can be formed by a combination of analogue filtering with additional digital filtering to optimize the shape of $h(t)$ and shorten the filter impulse response. For the purpose of this treatment we remain in the analogue domain, assuming a straightforward conversion to the digital domain as long as the sampling theorem is satisfied. The influence of the filter in the time-domain can be described by a convolution of the measurement signal with $h(t)$, shown in figure 1d).

Following the convolution we multiply the signal with the CDM decoding function $d(t-\tau)$, figure 1e) which is also periodic with $T_{\mathrm{c}}$ and dependent on the decoding delay $\tau$. Setting $\tau=t_{i}$ will ideally select the signal from source $i$. Apart from the code information, $d(t-\tau)$ additionally incorporates zero-valued grace periods of length $T_{\text {grace }}$ between the code symbols. This is intended to prevent symbol transition effects from causing inter-symbol interference, which can lead to both crosstalk of signals between range channels and non-linear SSB operation. For this to work $T_{\text {grace }}$ has to be larger than the filter impulse response length $T_{\mathrm{h}}$. Using the distributivity property of the convolution the filtered and spatially decoded signal $e(t, \tau)$ can now be described as:

$$
e(t, \tau)=d(t-\tau) \cdot A(\tau) \cdot \operatorname{Re}\left\{h(t) *\left(g(t-\tau) \cdot \exp \left[\mathrm{i}\left(s \cdot \varphi_{\mathrm{c}}(t-\tau)+\theta(\tau)\right)\right]\right)\right\}
$$

The signal $e(t, \tau)$ is also illustrated in figure 1f), where it can be seen that no symbol transition effects enter the signal due to the inclusion a the sufficiently long grace period $T_{\text {grace }}$. However, if $T_{\text {grace }}$ is not chosen long enough, several signal sources could influence the signal in (5) and $A(\tau)$ and $\theta(\tau)$ are now a function of $\tau$ to reflect this.

To derive the extended SSB equations we follow a similar complex Fourier series expansion of (5) as described in $[21,22]$, employing the assumption of stationary phase $\theta_{i}$. In our case this additionally requires 
using the distributivity property of the convolution and the assumption that the filter impulse response length $T_{\mathrm{h}}$ is smaller than the code repetition frequency $T_{\mathrm{c}}$. We can then conclude with the following extension of (1) and (2), where the band pass filtered signal $e_{n}(t, \tau)$, filtered with bandwidth $\pm 0.5 \cdot f_{\mathrm{c}}$ around the chosen carrier harmonic of order $n$, is given by:

$$
e_{n}(t, \tau)=A(\tau) \cdot \operatorname{Re}\left\{c_{n}(\tau) \cdot \exp \left[\mathrm{i}\left(2 \pi n f_{\mathrm{c}} t+\theta(\tau)\right)\right]+c_{-n}(\tau) \cdot \exp \left[\mathrm{i}\left(-2 \pi n f_{\mathrm{c}} t+\theta(\tau)\right)\right]\right\}
$$

The corresponding complex coefficients $c_{ \pm n}(\tau)$, which now incorporate the effects of the filter impulse response, any optional intensity modulation and the decoding with the pseudo-random code, are given by:

$$
c_{n}(\tau)=\frac{1}{2 \pi} \int_{-\frac{T_{\mathrm{c}}}{2}}^{\frac{T_{\mathrm{c}}}{2}} d(t-\tau) \cdot \exp \left[-\mathrm{i} 2 \pi n f_{\mathrm{c}} t\right] \cdot \int_{-\frac{T_{\mathrm{c}}}{2}}^{\frac{T_{\mathrm{c}}}{2}} h\left(t-t^{\prime}\right) \cdot g\left(t^{\prime}\right) \cdot \exp \left[\mathrm{i}\left(s \cdot \varphi_{\mathrm{c}}\left(t^{\prime}\right)\right] d t^{\prime} d t\right.
$$

Here, the inner integral is the circular convolution with $h(t)$ [27], which unlike the regular convolution is periodic in $T_{\mathrm{c}}$. The transition between regular and circular convolution is straightforward in this case because $T_{\mathrm{h}}<T_{\mathrm{c}}$. Note that both the final signal $e_{n}(t, \tau)$ and the coefficients $c_{ \pm n}(\tau)$ now depend on the decoding delay $\tau$. If $\tau=t_{i}$ and no inter-symbol interference occurs then $\theta(\tau)=\theta_{i}$ and $A(\tau)=A_{i}$ and the desired interferometric phase $\theta_{i}$ of source $i$ can be demodulated from $e_{n}(t, \tau)$ via multiplication of a complex carrier at frequency $f_{\mathrm{wf}}$. If inter-symbol interference occurs a mixture of signals from different sources might be demodulated and the sources cannot be completely separated. For the remainder of this paper the demodulated signal based on (6) will be referred to as the quadrature signal.

It is worth noting that using (6) and (7) the effect of an anti-aliasing filter (if $T_{\mathrm{h}}<T_{\mathrm{c}}$ ) on any type of periodic phase and amplitude modulation function can be analyzed. This includes intensity modulation CDM [14-16] by setting $\varphi_{\mathrm{c}}(t)=0$ and modulating $g(t)$ instead. Digitally enhanced heterodyne/homodyne interferometry $[17,18]$ can also be analysed by modulating $s \cdot \varphi_{\mathrm{c}}(t)$ with the phase step coding used there. In this case, if a heterodyne carrier is to be modelled, the phase shift by the heterodyne carrier has to be an integer multiple of $2 \pi$ within $T_{\mathrm{c}}$.

It is important to keep in mind that the autocorrelation properties of m-sequence coding, described in section 2.2, are not, in general, valid when the assumption of stationary phase $\theta_{i}$ is dropped. Also the Fourier expansion of the SSB equations that was used to derive (6) and (7) from (5) made use of the assumption of a stationary phase $\theta_{i}$. While the general validity of (6) and (7) for regular low-frequency signal appears to be given, it has been noted that interferometric high-frequency signals can be folded back into the base signal band of other range channels by higher order carriers of the SSB carrier comb. This has been indicated by a simple qualitative computer model that is not included here for brevity and is believed to be the reason for the noise behaviour described in section 4.1. Further theoretical work will be needed to extend the theory to nonstationary $\theta_{i}$. 


\section{Experimental Setup and Signal Processing}

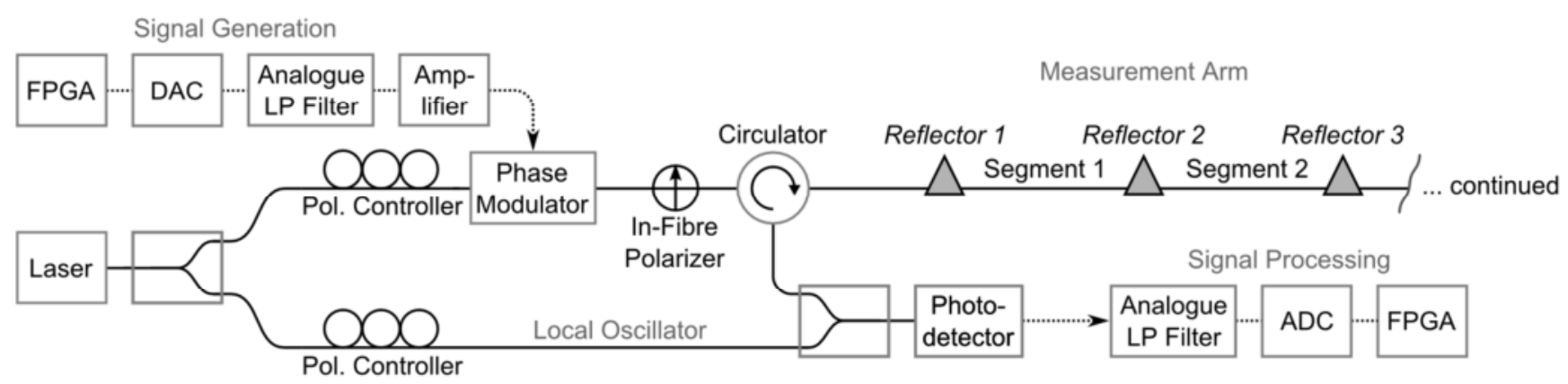

Figure 2. Optical setup in optical fibre based Mach-Zehnder interferometric configuration.

\subsection{Experimental setup}

Figure 2 illustrates the optical setup used for the experiments. Light from a $1550 \mathrm{~nm}$ single mode diode laser (Eblana Photonics EP1550-NLW-B; Linewidth: $200 \mathrm{kHz}$; Power: $10 \mathrm{~mW}$ ) enters a Mach-Zehnder interferometer constructed using standard SMF-28 type optical fibre. In one arm, a polarization controller [28] ensures that the light is linearly polarized and aligned with the principle axis of the electro-optic phase modulator (Photline MPZ-LN-10; Bandwidth: $10 \mathrm{GHz}$ ). This is necessary because of the inherent dependence of the voltage-to-phase-modulation transfer function on the polarization direction in lithium niobate modulators [29]. An in-fibre polarizer, aligned to the same polarization axis, is inserted after the phase modulator to filter out any remaining light from the unwanted polarization direction. We find that only the combination of polarization controller and polarizer allows for drift free and stable operation. The polarization direction is set by finding the operating point where linear operation is achieved, as will also be discussed in section 4.1. The operating point is dependent on both polarization direction and scaling factor $s$ of the phase modulation waveform $\varphi_{\mathrm{c}}(t)$. The phase modulator is driven by a waveform generated by the Field Programmable Gate Array (FPGA) followed by a digital-to-analogue converter (DAC), anti-aliasing filter and driving amplifier, details of the signal generation are discussed further in section 3.2.

From the phase modulator and in-fibre polarizer the light is guided to the measurement arm by an optical circulator. Here, equidistantly placed weak reflectors provide the signals measured by this scheme, the construction and placement of the reflectors is discussed in section 3.3. After the light has returned from the reflectors in the measurement arm it is guided by the circulator to the photo detector where it is mixed with light from the local oscillator (LO) arm and further signal processing, described in section 3.4 occurs. A second polarization controller in the LO arm is inserted to prevent polarization-induced signal fading in the interferometer. While, in principle, the scheme is insensitive to variations in signal strength because both components of the quadrature signals will be affected equally, complete signal failure can obviously not be dealt with and needs to be prevented. We assume that the light returning from the measurement arm remains largely linearly polarized. In this case, if the polarization controller in the LO is set to obtain a circular polarization state at the final coupler just before the photo detector, no complete fading can occur.

\subsection{Signal generation}

As shown in figure 2, the FPGA (Altera Cyclone IV on Terasic DE2-115 board), in conjunction with a data acquisition daughter board containing the analogue-to-digital converter (ADC) and DAC, implements both the signal generation and signal processing. Both the DAC and the ADC currently run at a clock frequency $f_{\mathrm{cl}}$ of $f_{\mathrm{cl}}=150 \mathrm{MHz}$ and are synchronous to each other, with appropriate anti-aliasing filters 
inserted on the analogue side of the converters. For signal generation the signal waveform that was transmitted to the DAC was pre-shaped electronically to counteract the non-ideal frequency response of the signal generation chain. This required the measurement of the combined impulse response of the analogue filter and amplifier, but has proved to increase the fidelity of the output waveform markedly.

An individual SSB waveform $\varphi_{\mathrm{wf}}(\mathrm{t})$ consists of $a=8$ samples and thus the SSB waveform frequency $\mathrm{f}_{\mathrm{wf}}$ is given by $\mathrm{f}_{\mathrm{wf}}=f_{\mathrm{cl}} \cdot a^{-1}=18.75 \mathrm{MHz}$. In this implementation $\varphi_{\mathrm{wf}}(t)$ is chosen to be of the shape $\varphi_{\mathrm{wf}}(t)=B\left(f_{\mathrm{wf}} t\right) \cdot \sin \left(2 \pi f_{\mathrm{wf}} t\right)$, where $B(t)$ is the Blackman window function [27]. This particular waveform shape can also be inspected in figure 1a). The code symbol length $T_{\text {symb }}$ is then given by:

$$
T_{\mathrm{symb}}=k \cdot T_{\mathrm{wf}}=k \cdot a \cdot f_{\mathrm{cl}}^{-1}
$$

In this implementation a total of $k=3$ individual SSB waveforms form a single code symbol and $T_{\text {symb }}=160 \mathrm{~ns}$. Using an m-sequence with $m=15$ code symbols allows the spatial separation of the same number of range channels and leads to a code with a repetition frequency of $f_{\mathrm{c}}=\left(\mathrm{m} \cdot T_{\mathrm{symb}}\right)^{-1}=417 \mathrm{kHz}$.

The required scaling factor $s$ for the phase modulation waveform $\varphi_{\mathrm{c}}(t)$ of the whole code, incorporating the m-sequence coding and based on the individual SSB waveform $\varphi_{\mathrm{wf}}(t)$ defined before, can be found by evaluating (7) at a harmonic index of $n=m * k=45$. This corresponds to $n$ individual SSB waveforms that make up one iteration of the code. The coefficients $c_{ \pm 45}$ are plotted in figure 3a) and a scaling factor of $s=0.65 \pi$ is chosen where coefficient $c_{+45}$ reaches zero and linear operation should occur. In practice, the calculated value of $s$ can only give a rough indication of the value and $s$ has to be manually tuned before the experiment by increasing the amplitude of the waveform that is generated in the FPGA. However, as described in section 3.1, when the polarization direction of the phase modulator was suitably stabilized, the value of $s$ was robust enough not to require any further tuning after the initial setting.
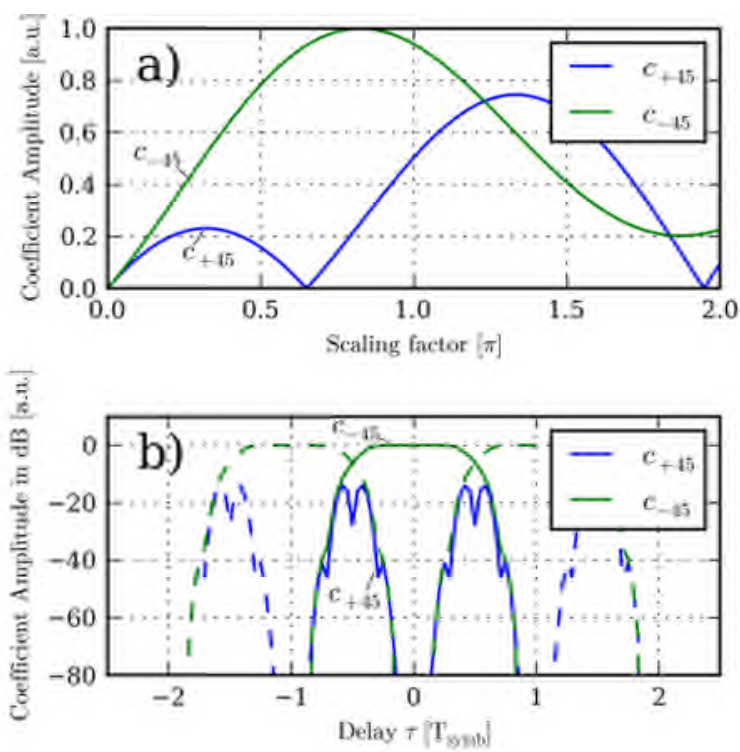

Figure 3. The dependence of the coefficients $c_{ \pm 45}$ on the scaling factor $s$ for a delay $\tau=0$ of the phase modulation function is shown in a). A working point of $s=0.65 \pi$ is chosen where $c_{+45}$ reaches zero. In b) the dependence of $c_{ \pm 45}$ on the decoding delay $\tau$ is shown for a fixed scaling factor of $s=0.65 \pi$. The dashed versions to the left and right of the graph are shifted by $\pm T_{\text {symb }}$ to highlight that there is no signal overlap at the designed reflector separation. 


\subsection{Construction and placement of reflectors in the measurement arm}

In figure $3 b)$ the dependence of the coefficients $c_{ \pm 45}$ in regard to the decoding delay $\tau$ is shown. The solid lines in figure $3 \mathrm{~b}$ ) show the coefficients for a reflector at a position corresponding to zero time-of-flight. It can be seen that both coefficients $c_{ \pm 45}$ are zero for decoding delays $|\tau|>T_{\text {symb }}$. For comparison, the dashed lines correspond to reflectors positioned at times-of-flights $\pm 1 \cdot T_{\text {symb }}$. It can be seen that if the reflectors are correctly positioned with an equidistant spacing, in theory, no signal energy will enter the signals corresponding to other range channels. Also it can be observed that there is some tolerance in the positioning of the reflectors. The standard segment length $l_{\mathrm{s}}$, corresponding to a time-of-flight of length $T_{\text {symb }}$, given by (8), to the reflector and back in fibre with a core index of refraction of $n_{\text {core }}=1.45$, will then be given below and in our case this equates to a segment length of $l_{\mathrm{s}}=16.5 \mathrm{~m}$.

$$
l_{\mathrm{s}}=\frac{c}{2 \cdot n_{\mathrm{core}}} \cdot T_{\mathrm{symb}}=1.03 \cdot 10^{8} \cdot T_{\mathrm{symb}}
$$

In CDM, neighbouring reflectors need to be placed equidistantly on a grid with a separation corresponding to the time-of-flight of one code symbol length $\mathrm{T}_{\text {symb }}$, given by (8). In general, there exists a common offset delay for all range channels that is caused by the time-of-flight in the lead length of the measurement arm and by signal processing delays and which can easily be accounted for by adjusting the decoding delays in the FPGA signal processing. It is assumed that there are no other fundamental limitations influencing the minimally achievable reflector separation $l_{\mathrm{S}}$ in (9) apart from the signal processing clock frequency $f_{\mathrm{cl}}$ in (8). The minimum reflector separation $l_{\mathrm{s}}$ is thus inversely proportional to $f_{\mathrm{cl}}$ when factors $k$ and $a$ are left unchanged in (8). The maximum number of reflectors that can be addressed in this scheme is given by the length $m$ of the $\mathrm{m}$-sequence used, where the maximum number of segments that can be evaluated is then $m-1$. Range channels where no reflector is placed do not produce a signal and are of no concern.

The scheme relies on the assumption that the reflectivities of the reflectors are sufficiently small not to substantially reduce the incident light of other reflectors or cause interference effects between light from different reflectors. When these assumptions cannot be maintained and a large number of reflectors need to be multiplexed, a more detailed power budget and reflectivity analysis as in [2] needs to be employed, which is beyond the scope of this paper. It should be noted, however, that in a Mach-Zehnder setup such as the one used here, the reflected signals are interferometrically amplified by the LO and thus the reflectivity of the infibre mirrors can be relatively low. Nevertheless, apart from signal-to-noise consideration, the signals from the reflectors also need to be considerably stronger than the underlying, distributed Rayleigh scattering in the fibre for the scheme to work. As can be seen from the experimental results shown in figure 7a), which are further discussed in section 4.1, the scheme in its current implementation can be expected to work well down to reflectivities of at least $-45 \mathrm{~dB}\left(3 \cdot 10^{-3} \%\right)$.

In this experiment, we use pairs of FC/PC connectors that are slightly detuned to achieve weak back reflections. This approach is only feasible in a lab environment due to its instability and the extensive tuning it requires. For future implementations, we propose using weak, broadband FBGs that are all centred at the same wavelength or in-fibre reflectors. In-fibre reflectors have been produced in the past by etching an air cavity in a fibre tip before resplicing the fibre [30] or by laser writing of Fabry-Perot cavities [31] into the fibre core. Furthermore, laser drilling of holes [32] and laser writing of microvoids [33] should also be adaptable to produce suitable in-fibre reflectors. In our opinion, laser writing techniques have potential to produce reflectors for sensing fibres in a roll-to-roll production process and without the need to break, resplice and recoat the fibre. This would permit very cost-effective and mechanically stable sensing fibre leads. 


\subsection{Signal processing}

The signal processing is illustrated in figure 4). After photo detection, the electronic signal is filtered with an anti-alias filter, digitized using an ADC and transmitted to the FPGA. Here further digital filtering results in the combined analogue and digital filter response $h(t)$, which is designed to have the characteristic of a band pass filter centred at the SSB waveform frequency $\mathrm{f}_{\mathrm{wf}}$. Additionally, $h(t)$ is required to have a length $T_{\mathrm{h}}$ that is smaller than the grace period of length $T_{\text {grace }}=2 \cdot T_{\text {wf }}$ to prevent inter-symbol interference, as shown in figure 1e) and described in section 2.3. To obtain a close approximation of the desired filter impulse response in practice, the actual impulse response of the analogue filter was measured and the digital part of the filter additionally pre-shaped to compensate for the non-ideal analogue filter frequency characteristics.

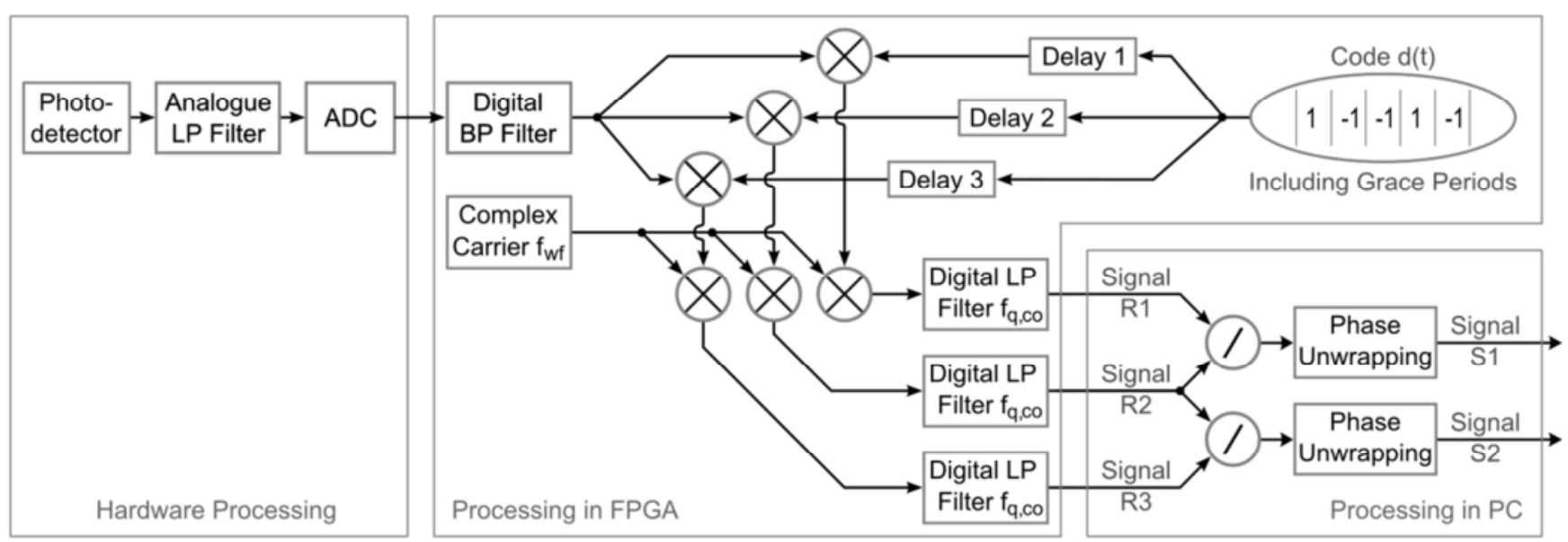

Figure 4. Outline of signal processing illustrated for three range channels. After hardware processing the incoming digital signal enters the FPGA. Here, decoding, demodulation and final low-pass filtering occurs for each of the three range channels R1 to R3. After being transmitted from the FPGA to a PC, the quadrature signals of R1 to R3 are subject to a complex division and phase unwrapping, which results in the phase difference signals $\Delta \theta$ for segments $\mathrm{S} 1$ and $\mathrm{S} 2$.

As can be seen in figure 4), the digitised and filtered signal is multiplied with delayed versions of the decoding function $d(t-\tau)$, discussed in section 2.3. Each delay corresponds to the time-of-flight to a specific reflector. As discussed in section 2.2, in unipolar-bipolar m-sequence coding the autocorrelation for code iterations with different delays is zero and out-of-range signals will cancel. Thus, in theory, only the signal energy from the reflector at the desired range, i.e. with the correct delay, will accumulate in each range channel. This spatially decoded signal corresponds to the signal shown in figure 1f) and the signal $e_{n}(t, \tau)$ given by (6).

Following this spatial decoding, the phase signals of each range channel are demodulated by multiplication with the complex carrier at the frequency of the SSB waveforms $f_{w f}$ and subsequent digital low-pass filtering with quadrature signal cut-off frequency $f_{\mathrm{q}, \mathrm{co}}$. Care has to be taken to demodulate a positive carrier harmonic $c_{+n}$ with a positive complex frequency and vice versa to obtain meaningful results. To prevent down-conversion of the signal from higher-order harmonics of the SSB carrier comb to the base band, the maximally permissible quadrature signal cut-off frequency $f_{\mathrm{q}, \max }$, using $T_{\text {symb }}$ given in (8), is limited to:

$$
f_{\mathrm{q}, \max }=0.5 \cdot f_{\mathrm{c}}=0.5 \cdot\left(m \cdot T_{\mathrm{symb}}\right)^{-1}
$$


Thus $f_{\mathrm{q}, \max }=208 \mathrm{kHz}$ is the theoretical maximum frequency that can be resolved with the current signal processing parameters. However, due to noise, which can cause spurious phase unwrapping, a much lower value of $f_{\mathrm{q}, \mathrm{co}}=20 \mathrm{kHz}$ has been chosen as the filter cut-off frequency to provide enough noise averaging. This will further discussed in section 4.1.

In our current implementation the filtered quadrature signals are transferred from the FPGA to a PC at this point, where a choice of evaluating reflector or segment data is made, however this could also be performed by the FPGA. For evaluating segment data the phases of two range channels are subtracted by complex division of the quadrature signals. Only then is the phase unwrapping [34] performed and the final phase difference $\Delta \theta$ along each fibre segment found. 


\section{Results and Discussion}

\subsection{Linearity, noise and measurements}

To analyse the linearity and noise properties of the system, the measurement arm configuration, illustrated in figure 5a) for evaluating range channel R3, was used. In this case, the connectors at range channels R1 and R2 are in contact and do not give a back reflection, while at range channels R4 no reflector is present. Thus, only the signal from the fibre-end reflection at range channel R3 is present and the linearity of its signal and its contribution on to the noise levels in the other range channels is examined in this experiment. The location of the fibre-end reflector can also be varied accordingly from range channel R1 to R4. The test signal for this experiment is produced by laser injection current modulation of the source.

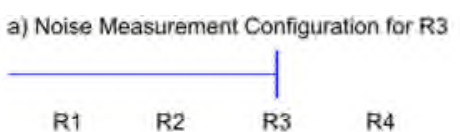

b) Crosstalk Measurement Configuration

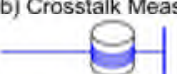

R1 P2T R2 R3
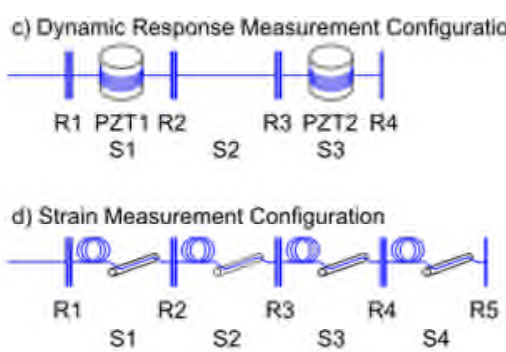

e) Strain Measurement Sensor-Arrangement

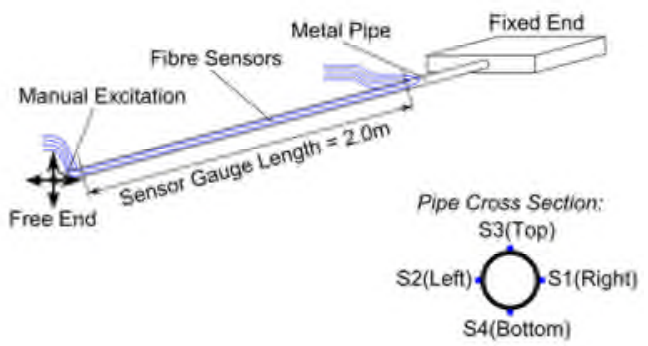

Symbols used. - f FC/PC connectors close (weak back reflection)

- Fibre end reflection

Figure 5. Measurement arm configurations used for the measurement of a) noise and linearity, b) cross-talk, c) dynamic response and d) strain measurement. e) illustrates how the sensors of configuration d) are attached to a vibrating metal pipe as test strain measurement object. Range channels, separated by $l_{\mathrm{s}}=16.5 \mathrm{~m}$ are labelled using the notation

$\mathrm{Rn}$ and the corresponding fibre segments using $\mathrm{Sn}$.

To analyse the linearity of the measurements the quadrature signals of a given range channel can be plotted in the complex plane. The resultant shape corresponds to a Lissajous ellipse which converges into a circle if linear operation is achieved [35]. Typical Lissajous figures for reflectors at range channels R1 to R4 are shown in figure 6). The scatter plots track the end point of all complex phasors that occur over the course of a measurement. The continuous line is the angular average of all the points within 120 angular sectors. 


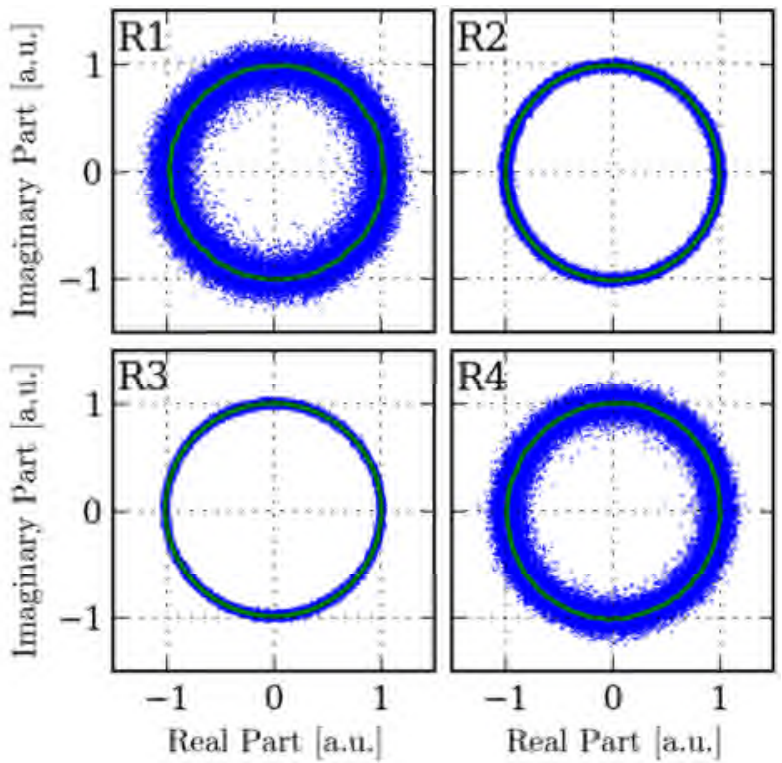

Figure 6. Scatter plot of the real and imaginary part for reflectors at range channels R1 to R4. The mean radius in each plot is normalized and the continuous line is the angular average over 120 angular sectors.

Using a Fourier transform over the averaged angular sector data, a quick measure of the degree of nonlinearity or ellipticity is given by the amplitude of the second harmonic in the transform. This was also the quantity used to experimentally tune the scaling factor $s$ of the phase modulation function $\varphi_{c}(t)$ described in section 3.2. The shapes of the ellipses shown in figure 6) were fitted to the coefficient ratio $c_{ \pm 45}$ in equation (6). The extracted values for the coefficient ratios are $1.5 \%, 2.0 \%, 0.9 \%$ and $2.5 \%$ for R1, R2, R3 and R4 respectively. Further analysis employing a simple computer simulation reveals that these coefficient ratios correspond to cyclic error amplitudes of $15 \mathrm{mrad}, 20 \mathrm{mrad}, 9 \mathrm{mrad}$ and $25 \mathrm{mrad}$ for R1, R2, R3 and R4 respectively.

As well as providing a measure of linearity, the Lissajous figure also yields information about the noise in the quadrature signals. From figure 6) it can be seen that amplitude noise is present and that it varies considerably depending on the position of the reflector in range channels R1 to R4. Furthermore, the observation has been made that spurious phase unwrappings are likely to occur when the scatter due to the amplitude noise is higher than the means radius and the hole in the Lissajous figure scatter plot starts being obscured. A single spurious phase unwrapping can lead to a permanent error in the phase signal of $\pm 2 \pi$ that cannot be corrected. If the noise levels are high enough that spurious phase unwrappings occur, the quadrature signals have to be averaged further to reduce the amplitude noise sufficiently. As was already discussed in section 3.4, this was the reason that the measurement bandwidth had to be reduced more than tenfold to $f_{\mathrm{q}, \text { co }}=20 \mathrm{kHz}$.

In addition to the variation of the amplitude noise with reflector position when a single source is present, as evident in the Lissajous figures in figure 6), it was also observed in other experiments with multiple signal sources that the amplitude noise level in any one range channel is dependent on the signal strength of sources present in other range channels. This leads to the conjecture that there is also some leakage of amplitude noise from the originating source to other range channels.

To thoroughly investigate the origin of the amplitude noise and any possible leakage in the quadrature signals a series of single reflector measurements with adjustable return signal levels was conducted. This experiment was also performed using the setup shown in figure 5a) and employs a single fibre-end reflector at 
range channel R3 as the signal source. In this experiment, the effective reflectivity of the signal source was successively lowered by inducing bend loss in the fibre lead, ranging from $-15 \mathrm{~dB}(3.3 \%)$ for the fibre-end Fresnel reflection without additional attenuation to $-57 \mathrm{~dB}\left(2 \cdot 10^{-4} \%\right)$ effective reflectivity. The spatial variation of the average root-mean-square (rms) signal amplitude over an $80 \mathrm{~m}$ range at each of the four reflectivity levels was recorded and is shown in figure $7 \mathrm{a}$ ).

It can be observed in figure 7a) that for each reflectivity level, outside of the range window surrounding the reflector, there is a mostly flat baseline of noise leakage. By additionally evaluating the amplitude noise in the Lissajous figure of the peak signal, it was verified that the level of rms amplitude noise there is the same as the level of rms amplitude noise in the baseline in figure 7a). Thus the amplitude noise appears to be evenly distributed over all range channels. All the plots were normalized to the corresponding peak amplitude. For the measurements from $-15 \mathrm{~dB}$ to $-45 \mathrm{~dB}$, the ratio between peak amplitude and baseline can be seen to remain largely constant. Only at $-57 \mathrm{~dB}$ does this relationship start to break down. This indicates that the strength of noise leakage is proportional to the strength of the signal source that causes the leakage. Most noise sources like LO shot noise, laser intensity noise, detector and signal processing noise would be expected to contribute a constant noise level regardless of the level of reflectivity. Any constant noise contribution of this kind would quickly increase in magnitude compared to the main peak when the reflectivity level of the main peak is lowered. Such behaviour is only starting to become visible at very low reflector strengths, i.e. $-57 \mathrm{~dB}$. This leaves only noise sources that are proportional to the source signal amplitude as a plausible cause for the observed amplitude noise. These types of noise could be shot noise from the reflector signal, direct signal crosstalk or laser phase noise that is outside the signal band and can thus be redistributed into other range channels as discussed in section 2.3.
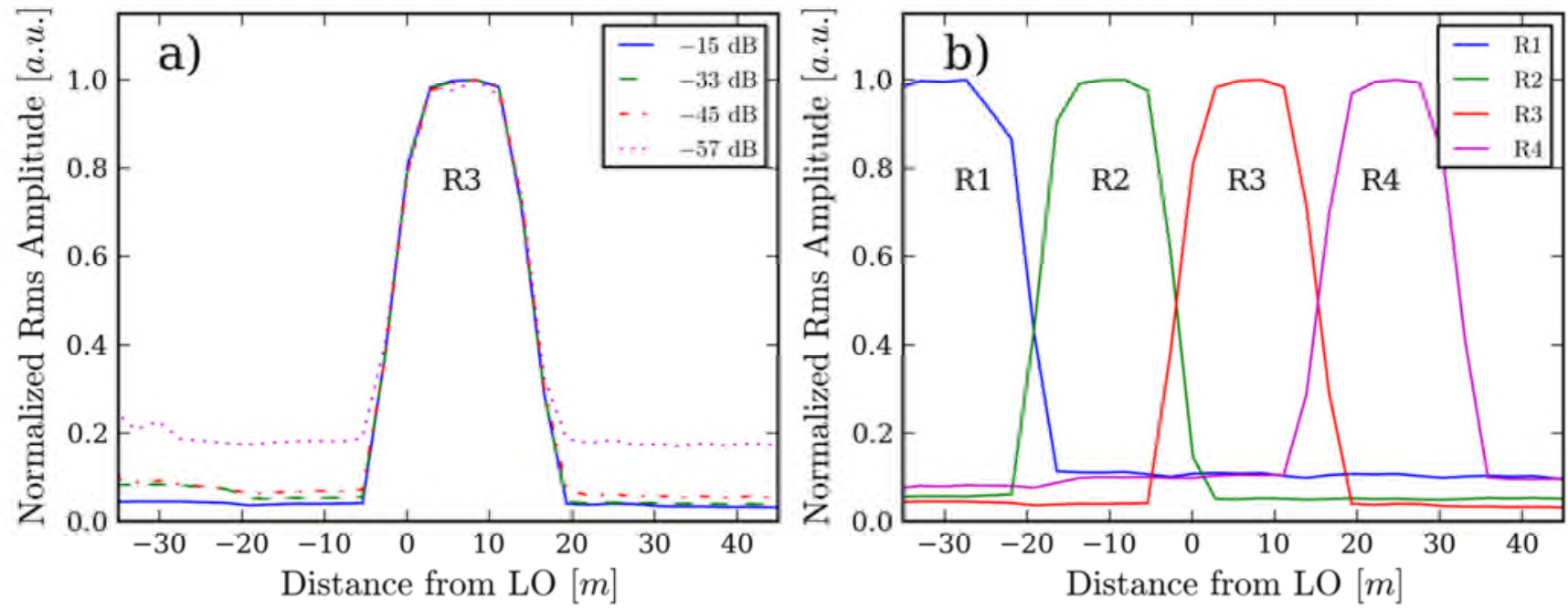

Figure 7. a) shows measurements of the average rms signal amplitude originating from one reflector kept at the same location for four different reflectivities. b) shows four rms amplitude measurements all taken at a constant reflectivity of $-15 \mathrm{~dB}$, but for four different locations at range channels $\mathrm{R} 1$ to $\mathrm{R} 4$ with varying distance from the LO balancing point. All measurements in a) and b) were normalized to the corresponding peak amplitude.

To further differentiate between the possible types of noise another experiment with the same singlereflector setup of figure 5a) was used. This time the reflector location was varied in respect to the LO balancing point, while the reflectivity was kept constant at $-15 \mathrm{~dB}$. Figure $7 \mathrm{~b}$ ) shows the graphs for four reflectors in range channels $\mathrm{R} 1$ to $\mathrm{R} 4$. It can be noted that the height of the baseline is dependent on the distance of the signal source from the LO balancing point. Reflectors in range channels R2 and R3, which are 
placed close to the LO balancing point, produce a noise floor approximately half that of reflectors in range channels R1 and R4, which are located further away. This result also agrees well with the perceived noise level in the Lissajous figures shown in figure 6). This leads to the conclusion that the bulk of the observed amplitude noise is dependent on the distance to the LO balancing point of the source that causes the leakage. Of the three possible types of noise mentioned previously only laser phase noise that was redistributed into other range channels would show this type of behaviour. The options available to mitigate the amplitude noise are further discussed in section 4.4.

While it has been established that direct signal crosstalk from one range channel to the other is not the main cause of the amplitude noise observed, we nevertheless measured the signal crosstalk levels in the quadrature signals in order to compare the different $\mathrm{m}$-sequence coding arrangements that were discussed in section 2.2. To this end we used the setup shown in figure $5 \mathrm{~b}$ ), where a piezoelectric fibre stretcher (PZT) produces a sinusoidal test signal of frequency $70 \mathrm{~Hz}$. The quadrature signals corresponding to this phase signal are a series of Bessel harmonics. The test signal is measured by evaluating the signal from the fibre-end reflector located in range channel R2. Signals registered at range channels R1 and R3, which are located at a distance corresponding to one segment length $\pm l_{\mathrm{s}}=16.5 \mathrm{~m}$, from the fibre-end and that possess no signal source of their own, are evaluated for the existence of signal crosstalk from R2.
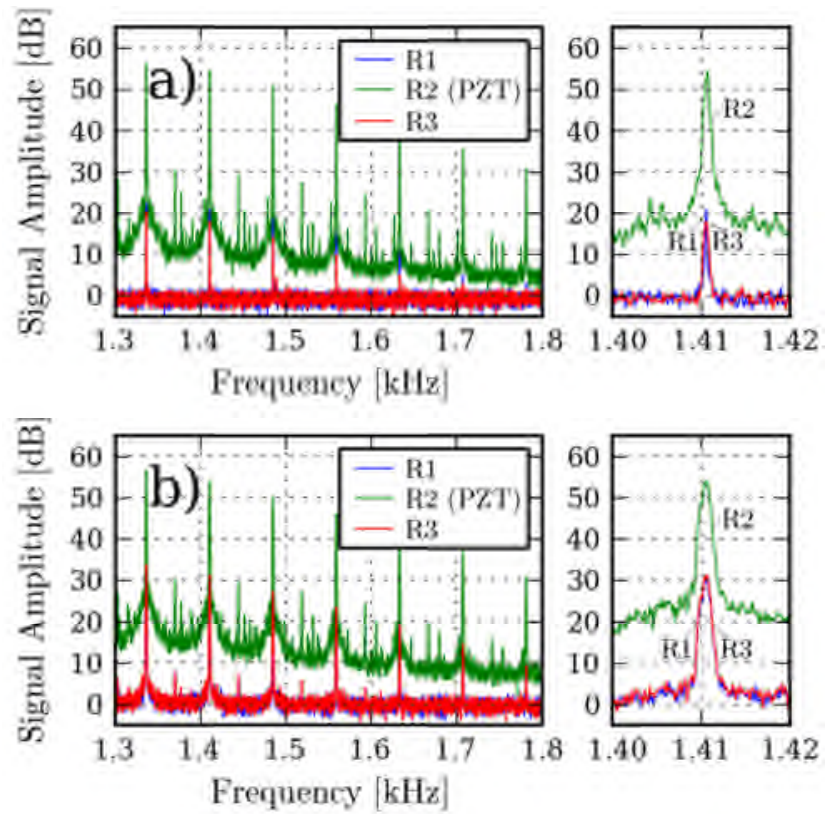

Figure 8. Measurement of signal crosstalk in quadrature signals from range channel R2 into neighbouring range channels R1 and R3. a) unipolar-bipolar m-sequence coding with $m=15$. b) bipolar-bipolar m-sequence coding with $m=15$. In both a) and b) the graphs on the right show an enlarged version of the peaks at $1.41 \mathrm{kHz}$.

Figure $8 \mathrm{a}$ ) and $8 \mathrm{~b}$ ) show the results of the crosstalk measurements for a frequency region between $1.3 \mathrm{kHz}$ and $1.8 \mathrm{kHz}$. In figure 8a), the measurement for unipolar-bipolar m-sequence coding with $m=15$ code symbols is shown, which is the coding arrangement that was used throughout this work. For all Bessel harmonics present, a nearly constant crosstalk level of $-32 \mathrm{~dB}$ for range channel R1 and of $-35 \mathrm{~dB}$ for R3 can be observed. In measurements not shown here, the crosstalk levels for range channels that are $\pm 2 l_{\mathrm{s}}$ or further from the signal source was found to be approaching $-48 \mathrm{~dB}$. This indicates that the length of the antialiasing filter impulse response $T_{\mathrm{h}}$ exceeds $T_{\text {grace }}$ in the current implementation of the signal processing, and the theoretically very high level of crosstalk amplitude suppression given in (3) for $R_{\mathrm{ub}}$ has not yet been fully 
realized. Figure $8 \mathrm{~b}$ ) shows the signals for bipolar-bipolar $\mathrm{m}$-sequence coding with $m=15$. The crosstalk levels are found to be $-23 \mathrm{~dB}$, which is exactly as predicted for $R_{\mathrm{bb}}$ in (3). Since the crosstalk for the same number of code symbols is higher in bipolar-bipolar m-sequence coding, there is a clear advantage when using unipolar-bipolar coding instead. This gain should be higher if the filter properties of the system can be improved in future implementations.

To determine the signal crosstalk and noise in the unwrapped phase signals, as opposed to the crosstalk and noise in the quadrature signals, the experimental setup shown in figure 5c) was used. Here, the two PZTs in segments $\mathrm{S} 1$ and $\mathrm{S} 3$ provide sinusoidal test signals at $370 \mathrm{~Hz}$ and $570 \mathrm{~Hz}$ respectively, while segment $\mathrm{S} 2$ is not excited. In figure 9a) the time traces of the phase difference signals $\Delta \theta$ along fibre segments $\mathrm{S} 1$ to S3 are shown, while figure 9b) shows the corresponding spectra. In figure 9b) the base of the two main peaks are enlarged to highlight that no direct signal crosstalk higher than the noise floor is visible. Thus, crosstalk levels in the phase signals are found to be lower than $-60 \mathrm{~dB}$, which are therefore of the same magnitude as those found in Wuchenich et al. [20]. The fact that crosstalk levels lower than $-60 \mathrm{~dB}$ can be realized in the phase signal, while previously crosstalk levels up of $-32 \mathrm{~dB}$ were determined in the quadrature signals is a consequence of the non-linear migration properties of noise and crosstalk from the quadrature signals to the phase signals. This is because the contribution of a given noise or crosstalk phasor to the measured phase angle is non-linearly dependent on the length of the signal phasor to which it is added. Any crosstalk measurement in phase data is thus highly dependent on the source signal strength and is not an objective measure of crosstalk without further details on the source strength configuration. Only crosstalk measurements in the quadrature measurements provide an easy comparison of crosstalk levels between different coding arrangements and anti-aliasing filter settings. The exact mathematical description of the nonlinear noise migration behaviour of noise from quadrature into phase signals is subject to active research [36] and is beyond the scope of this paper.
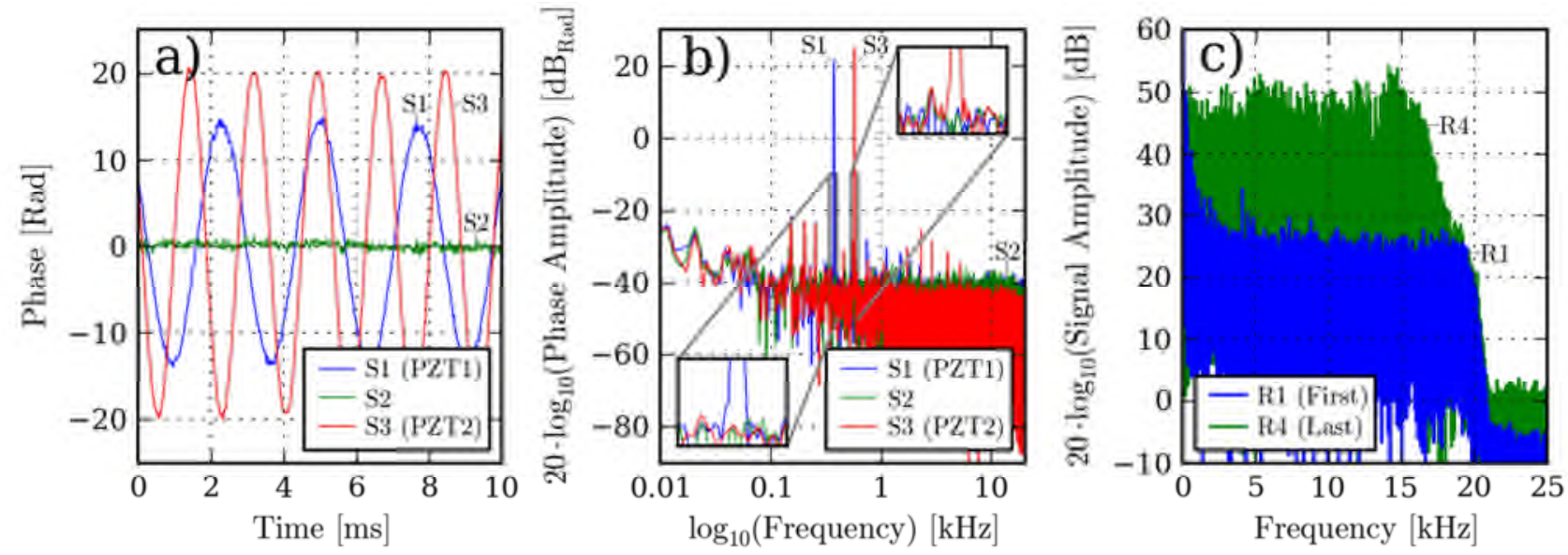

Figure 9. Measurement of the dynamic response of the system. a) shows the time traces of the phase signals of three fibre segments S1 to S3, where segments S1 and S3 were excited with sinusoidal waveforms at $370 \mathrm{~Hz}$ and $570 \mathrm{~Hz}$ respectively. b) shows the corresponding phase signal spectra with logarithmic frequency scale, where the region at the base of the main peaks is enlarged. c) shows the spectra of the corresponding quadrature signals for the first and last range channels used in this experiment.

To evaluate the resultant noise in the phase difference along a fibre segment $\Delta \theta$, a series of measurements with the PZTs switched off show that the typical rms phase noise level present in the segment data is $2.3 \mathrm{mrad} \mathrm{Hz} \mathrm{H}^{-0.5}$ in all segments regardless of segment position. These measurements are not shown here because similar noise characteristics are also evident in the results shown for segment S2 in figure 9a) and b). 
In figure 9b) segment $S 2$ shows a mainly white noise spectrum apart from a small noise increase at very low frequencies.

\subsection{Dynamic response measurements}

For the low-frequency, high-amplitude phase signals that this scheme is designed to measure, the spectral requirements of the quadrature signal for a given phase signal are best described by the rate of phase change that needs to be resolved. The maximum rate of change of the phase signals in segment S1and S3 can be extracted from figure 9a) to be $3.2 \cdot 10^{4} \mathrm{rad} \mathrm{s}^{-1}$ for segment S1 and $7.2 \cdot 10^{4} \mathrm{rad} \mathrm{s}^{-1}$ for segment S3. The furthest away range channel R4 carries contributions from all signal sources present in the experiment, as can be seen in figure $5 \mathrm{c}$ ). Thus, the signal of range channel R4 has to resolve a maximum rate of phase change of $\dot{\theta}_{\text {max }}=1.0 \cdot 10^{5} \mathrm{rad} \mathrm{s}^{-1}$ at points in time when the highest rates of phase change of both sinusoidal signals from the two PZTs coincide. As a first estimate of the spectral requirement the rate of phase change $\dot{\theta}_{\max }$ is equated to the maximum frequency requirement of the quadrature signal to yield a spectral requirement of $\dot{\theta}_{\max } \cdot(2 \pi)^{-1}=16 \mathrm{kHz}$. Figure $\left.9 \mathrm{c}\right)$ plots the spectrum of the quadrature signals of range channels R1 and $\mathrm{R} 4$. The spectrum of the first range channel R1, which does not experience any test signal, is inserted here as a measure of the noise floor and shows a mainly white noise behaviour with contributions from what is believed to be laser phase noise at the low-frequency end of the spectrum. At the high-frequency end of the spectrum for range channel R4, it can be seen that the spectral content does indeed begin to drop at a frequency of around $16 \mathrm{kHz}$. To allow for the full slope visible in figure 9c) to be resolved an additional safety factor of $25 \%$ of the spectral requirement seems reasonable. It can thus be summarized that the chosen quadrature signal bandwidth of $f_{\mathrm{q}, \mathrm{co}}=20 \mathrm{kHz}$ that is used in this implementation allows the resolution of phase change rates of $\dot{\theta}_{\text {max }}=1.0 \cdot 10^{5} \mathrm{rad} \mathrm{s}^{-1}$.

\subsection{Strain measurement}

Finally, the system was used to make exemplary strain measurements. The sensor configuration for this experiment is shown in figure 5d). Here, for each segment $\mathrm{S} 1$ to $\mathrm{S} 4$ a section of gauge length $\mathrm{l}_{\mathrm{g}}=2 \mathrm{~m}$ out of the total segment length $l_{\mathrm{s}}$ is used as a strain sensor, while the remainder of each fibre segment is rolled up. The strain sensing sections of the four segments are attached to four sides of a metal pipe that is fixed at one end but is free to vibrate on the other. The arrangement of the sensors on the pipe is also illustrated in figure 5e). This experiment is designed to obtain test signals of low frequency but with high signal amplitudes. Signals of these characteristics are expected to be encountered in many real applications of dynamic strain sensing on large engineering structures. 


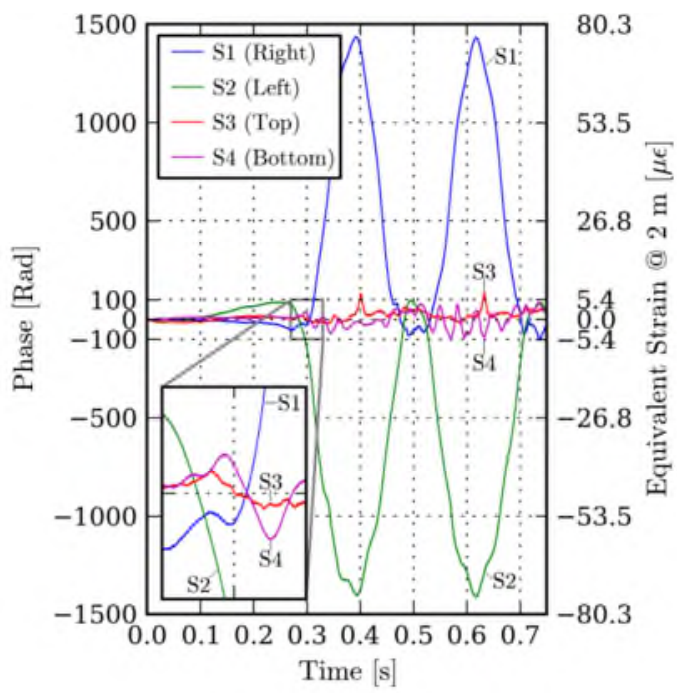

Figure 10. This graph shows a typical low-frequency, high-amplitude strain measurement for four long-gauge length strain sensors. The inset shows a zoomed region at the time of excitation over a time period of $60 \mathrm{~ms}$ and a phase range of $\pm 100 \mathrm{rad}$. The secondary axis shows the strain that is equivalent to this phase signal at a sensor gauge length of $2 \mathrm{~m}$.

An exemplary measurement using this configuration with a horizontal excitation of the metal pipe is shown in figure 10). It can be clearly seen that both sensors in segments S1 and S2 attached to the left and right side of the pipe show a high amplitude vibration, while the sensors at segments S3 and S4 attached to the top and bottom of the pipe show only little reaction to this excitation. The high measurement quality can be inspected in the zoomed inset. The strain of the fibre that these phase changes correspond to is shown on the secondary axis. The strain $\varepsilon$ can be calculated from the measured phase difference $\Delta \theta$ using the following equation adapted from Kirkendall et al. [7]:

$$
\varepsilon=\frac{\Delta \theta \cdot \lambda}{2 l_{\mathrm{g}} \cdot 2 \pi \cdot n_{\text {core }} \cdot k}
$$

Here $\lambda$ is the wavelength of the laser light and $k$ is the strain sensitivity factor of the fibre. We take $k$ to be $k=0.795$, which is the value given by Roths et al. [37] for SMF-28 type fibre. An additional factor of two in the denominator is included here because in our setup the light passes the sensor gauge length $l_{\mathrm{g}}$ twice.

\subsection{Discussion}

In the experiments presented here we had to use the polarization control arrangement shown in figure 2 and discussed in section 3.1, using a polarization controller and an additional in-fibre polarizer, because no polarization-maintaining phase modulator was available. However, for future implementations we strongly recommend using polarization-maintaining fibre for the section connecting the laser diode to the phase modulator. This would eliminate the need for the polarization controller and the in-fibre polarizer and should prevent any drifts in polarization direction influencing the phase modulation strength and thus the linearity of the measurements. We also recognize that in a real-life application the absence of polarization-induced signal fading in the measurement arm cannot be guaranteed by simply using a polarization controller in the LO as discussed in section 3.1. Further options to mitigate polarization-induced signal fading in future implementations include using polarization-diversity detection [38] or employing polarization-maintaining fibre throughout the interferometer and the measurement arm. 
In section 4.1 the typical rms noise levels in the measured phase change along a fibre segment $\Delta \theta$ were

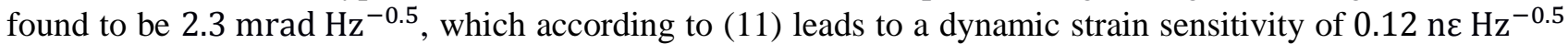
at a sensor gauge length $l_{\mathrm{g}}=2 \mathrm{~m}$. While the sensor gauge length characteristics between the sensing schemes might be different, the measured dynamic strain sensitivity of this scheme compares well with those for noninterferometric, short-gauge length FBG strain sensors, which are typically on the order of 0.5 to $10 \mathrm{n} \varepsilon \mathrm{Hz}^{-0.5}$ [39]. The maximum cyclic error was found to be $0.025 \mathrm{rad}$ in the current implementation. Using (11) and realizing that the systematic error in the range channel data can enter the segment data twice, the maximum systematic phase error can be given as $2.7 \mathrm{n} \varepsilon$. Because the magnitude of the cyclic error cannot exceed $0.5 \pi$ and is non-cumulative, this systematic error is bounded in magnitude to below $0.17 \mu \varepsilon$ even in a worst case scenario, however, the given values for the systematic errors obviously require the total absence of spurious phase unwrappings. It is also worth noting that strain noise and systematic errors scale inversely with the sensor gauge length, leading to even higher strain sensitivities for longer sensor gauge lengths. As in all fibre strain sensors there is a strong inherent sensitivity to temperature [40] that can negatively affect strain measurements and which has not been addressed so far in this scheme.

In section 4.2, a maximum phase change rate of $\dot{\theta}_{\max }=1.0 \cdot 10^{5} \mathrm{rad} \mathrm{s}^{-1}$ that can be resolved in the current implementation was calculated. This rate is shared by all the sensors present in the system, thus the total phase change rate has to be divided by the number of sensors if all sensors are expected to have similar strain signal characteristics. In the strain measurement configuration in figure $5 \mathrm{~d}$ ) with four strain sensors this equates to $\dot{\theta}_{\text {max }, \mathrm{s}}=2.5 \cdot 10^{4} \mathrm{rad} \mathrm{s}^{-1}$ for each sensor. Again, using (11) for $l_{\mathrm{g}}=2 \mathrm{~m}$, this results in a permissible strain change rate of $\dot{\varepsilon}_{\text {max }, \mathrm{s}}=1.3 \mathrm{m \varepsilon} \mathrm{s}^{-1}$ per sensor.

While the noise levels in the strain measurement were found to compare well with standard techniques used for strain measurement applications such as FBGs, the more important consequence of phase noise from the laser source is the reduction of the achievable quadrature signal cut-off frequency $f_{\mathrm{q}, \text { co }}$ and thus ultimately the reduction of the permissible strain change rate $\dot{\varepsilon}_{\max , \mathrm{s}}$. As discussed in section 4.1, the reduction is due to noise leakage originating from strong reflectors that can overshadow weak reflectors in other range channels and lead to spurious phase unwrappings there. In order to maintain practical reflectivity tolerances and polarization-induced fading allowances for the reflector setup, $f_{\mathrm{q}, \mathrm{co}}$ had to be lowered sufficiently to reduce the noise to an acceptable level through averaging, in this implementation by a factor of more than 10. Other steps to mitigate this noise behaviour without reducing $f_{\mathrm{q}, \mathrm{co}}$ are using laser diodes with narrower line widths or lowering the average distance to the LO of the reflectors present in the system. In the setup shown in figure $5 \mathrm{~d}$ ) the average distance to the LO could be lowered if the segment length $l_{\mathrm{s}}$ given by (10) could be reduced from its current value of $l_{\mathrm{s}}=16.5 \mathrm{~m}$, ideally down to the sensor gauge length $l_{\mathrm{g}}=$ $2 \mathrm{~m}$.

In order to reduce the fibre segment length, either for improving the spatial resolution or for lowering the average LO distance, the speed of the signal processing would need to be increased. Because the analogue bandwidths of both DAC and ADC are limited by analogue filters in a well-known fashion in this implementation, conclusions about the scaling of the signal processing parameters can be drawn. If the current clock frequency $f_{\mathrm{cl}}$ of $150 \mathrm{MHz}$ is scaled up by a factor of $x$ to $f_{\mathrm{cl}}=x \cdot 150 \mathrm{MHz}, l_{\mathrm{s}}$ will reduce to $l_{\mathrm{s}}=\frac{16.5 \mathrm{~m}}{x}$ according to (10). The theoretically possible maximum quadrature signal frequency $f_{\mathrm{q}, \max }$ of (9) will also increase when the signal processing is scaled up by $x$ to $f_{\mathrm{q}, \max }=3.125 \mathrm{MHz} \cdot \frac{x}{\mathrm{~m}}$, where the msequence length $m$ determines the maximum number of range channels that can be separated. In this context, signal generation and processing at many $\mathrm{GHz}$ clock speeds is state of the art in optical communications technology and has been demonstrated for optical measurement applications [41] as well. 


\section{Conclusions}

A novel signal processing scheme that combines code-division multiplexing with single-sideband homodyne signal processing scheme has been presented. The equations governing the scheme as well as the signal processing parameters have been described in detail. The scheme has been applied to fibre segment interferometry for strain sensing applications, where four long-gauge length strain sensors of gauge length $l_{\mathrm{g}}=2 \mathrm{~m}$ have been successfully multiplexed and dynamic strain sensitivities of $0.12 \mathrm{nc} \mathrm{Hz}^{-0.5}$ have been achieved.

The main limitation of the scheme in its current implementation is the leakage of high-frequency laser phase noise from the signal source into other range channels in the system. This has reduced the permissible strain change rate by more than a factor of ten to $1.3 \mathrm{mes}^{-1}$ per sensor. The dynamic parameters of the scheme could be improved using laser diodes with narrower line widths, or by lowering the average distance to the $\mathrm{LO}$ of the reflectors present in the system by scaling the clock frequency.

However, as the combined cost of all the equipment used in this prototype was less than $\$ 10 \mathrm{k}$ the possibility of a cost-effective, relative strain change measurement system has been demonstrated. Finally, the possibility exists to combine this technique with FBG sensors operating at other wavelengths to obtain both short-gauge and long-gauge length strain information from the same sensing fibre.

\section{Acknowledgment}

This work was supported by the UK Engineering and Physical Sciences Research Council (EPSRC). 


\section{References}

[1] Dakin, J. P., Wade, C. A. and Henning, M. (1984), "Novel optical fibre hydrophone array using a single laser source and detector", Electronics Letters, vol. 20, no. 1, pp. 53-54.

[2] Cranch, G. A. and Nash, P. J. (2001), "Large-scale multiplexing of interferometric fiber-optic sensors using TDM and DWDM", Journal of Lightwave Technology, vol. 19, no. 5, pp. 687-699.

[3] Butter, C. D. and Hocker, G. B. (1978), "Fiber optics strain gauge", Applied Optics, vol. 17, no. 18, pp. 2867-2869.

[4] Kersey, A. D., Davis, M. A., Patrick, H. J., LeBlanc, M., Koo, K., Askins, C., Putnam, M. and Friebele, E. J. (1997), "Fiber grating sensors", Lightwave Technology, Journal of, vol. 15, no. 8, pp. 1442-1463.

[5] Thévenaz, L. (2006), "Review and Progress on Distributed Fibre Sensing", Optical Fiber Sensors, Optical Society of America, .

[6] Bao, X. and Chen, L. (2011), "Recent progress in Brillouin scattering based fiber sensors", Sensors, vol. 11, no. 4, pp. 4152-4187.

[7] Kirkendall, C. K. and Dandridge, A. (2004), "Overview of high performance fibre-optic sensing", Journal of Physics D: Applied Physics, vol. 37, no. 18, pp. 197-216.

[8] Glisic, B. (2011), "Influence of the gauge length on the accuracy of long-gauge sensors employed in monitoring of prismatic beams", Measurement Science and Technology, vol. 22, no. 3.

[9] Carden, E. P. and Fanning, P. (2004), "Vibration based condition monitoring: a review", Structural Health Monitoring, vol. 3, no. 4, pp. 355-377.

[10] Barlas, T. and Van Kuik, G. (2010), "Review of state of the art in smart rotor control research for wind turbines", Progress in Aerospace Sciences, vol. 46, no. 1, pp. 1-27.

[11] Song, G., Sethi, V. and Li, H. N. (2006), "Vibration control of civil structures using piezoceramic smart materials: A review", Engineering Structures, vol. 28, no. 11, pp. 1513-1524.

[12] Glover, I. and Grant, P. M. (2009), Digital communications, 3rd ed, Pearson Education.

[13] Takeuchi, N., Sugimoto, N., Baba, H. and Sakurai, K. (1983), "Random modulation cw lidar", Applied Optics, vol. 22, no. 9, pp. 1382-1386.

[14] Al-Raweshidy, H. S. and Uttamchandani, D. (1990), "Spread spectrum technique for passive multiplexing of interferometric optical fiber sensors", Optics Communications, vol. 80, no. 1, pp. 18-22.

[15] Kersey, A. D., Dandridge, A. and Davis, M. A. (1992), "Low-crosstalk code-division multiplexed interferometric array", Electronics Letters, vol. 28, no. 4, pp. 351-352.

[16] Kullander, F., Laurent, C., Zyra, S. and Geis, H. (1998), "Crosstalk reduction in a code division multiplexed optical fiber sensor system", Optical Engineering, vol. 37, no. 7, pp. 2104-2107. 
[17] Shaddock, D. A. (2007), "Digitally enhanced heterodyne interferometry", Optics Letters, vol. 32, no. 22, pp. 3355-3357.

[18] Sutton, A. J., Gerberding, O., Heinze, G. and Shaddock, D. A. (2012), "Digitally enhanced homodyne interferometry", Optics Express, vol. 20, no. 20, pp. 22195-22207.

[19] De Vine, G., Rabeling, D. S., Slagmolen, B. J., Lam, T. T., Chua, S., Wuchenich, D. M., McClelland, D. E. and Shaddock, D. A. (2009), "Picometer level displacement metrology with digitally enhanced heterodyne interferometry", Optics Express, vol. 17, no. 2, pp. 828-837.

[20] Wuchenich, D. M. R., Lam, T. T. -., Chow, J. H., McClelland, D. E. and Shaddock, D. A. (2011), "Laser frequency noise immunity in multiplexed displacement sensing", Optics Letters, vol. 36, no. 5, pp. 672-674.

[21] Ostwald, O. and Schiek, B. (1981), "Network analysis by phase-modulated homodyne detection", IEEE Transactions on Instrumentation and Measurement, vol. IM-30, no. 2, pp. 152-154.

[22] Voges, E., Ostwald, O., Schiek, B. and Neyer, A. (1982), "Optical phase and amplitude measurement by single sideband homodyne detection", IEEE Journal of Quantum Electronics, vol. QE-18, no. 1, pp. 124-129.

[23] Dandridge, A., Tveten, A. B. and Giallorenzi, T. G. (1982), "Homodyne Demodulation Scheme for fiber Optic Sensors using phase generated carrier", IEEE Journal of Quantum Electronics, vol. 18, no. 10, pp. 1647-1653.

[24] Jackson, D. A., Kersey, A. D., Corke, M. and Jones, J. D. C. (1982), "Pseudoheterodyne detection scheme for optical interferometers", Electronics Letters, vol. 18, no. 25-26, pp. 1081-1083.

[25] Tatam, R. P., Jones, J. D. C. and Jackson, D. A. (1986), "Opto-electronic processing schemes for the measurement of circular birefringence", Optica Acta, vol. 33, no. 12, pp. 1519-1528.

[26] Dixon, R. C. (1984), Spread spectrum systems, 2nd ed, John Wiley \& Sons, Inc.

[27] Proakis, J. G. and Manolakis, D. G. (1996), Digital signal processing: principles algorithms and applications, 3rd ed, Pearson.

[28] Lefevre, H. (1980), "Single-mode fibre fractional wave devices and polarisation controllers", Electronics Letters, vol. 16, no. 20, pp. 778-780.

[29] Wooten, E. L., Kissa, K. M., Yi-Yan, A., Murphy, E. J., Lafaw, D. A., Hallemeier, P. F., Maack, D., Attanasio, D. V., Fritz, D. J. and McBrien, G. J. (2000), "A review of lithium niobate modulators for fiber-optic communications systems", Selected Topics in Quantum Electronics, IEEE Journal of, vol. 6, no. 1 , pp. 69-82.

[30] Cibula, E. and Donlagic, D. (2010), "Low-loss semi-reflective in-fiber mirrors", Optics Express, vol. 18, no. 11, pp. 12017-12026. 
[31] Rao, Y. J., Deng, M., Duan, D. W., Yang, X. C., Zhu, T. and Cheng, G. H. (2007), "Micro Fabry-Perot interferometers in silica fibers machined by femtosecond laser", Optics express, vol. 15, no. 21, pp. $14123-14128$.

[32] Van Brakel, A., Grivas, C., Petrovich, M. N. and Richardson, D. J. (2007), "Micro-channels machined in microstructured optical fibers by femtosecond laser", Optics Express, vol. 15, no. 14, pp. 8731-8736.

[33] Tomlins, P. H., Smith, G. N., Woolliams, P. D., Rasakanthan, J. and Sugden, K. (2011), "Femtosecond laser micro-inscription of optical coherence tomography resolution test artifacts", Biomedical optics express, vol. 2, no. 5, pp. 1319-1327.

[34] Itoh, K. (1982), "Analysis of the phase unwrapping algorithm", Applied Optics, vol. 21, no. 14, pp. 2470-2470.

[35] Heydemann, P. L. M. (1981), "Determination and correction of quadrature fringe measurement errors in interferometers", Applied Optics, vol. 20, no. 19, pp. 3382-3384.

[36] De Freitas, J. (2007), "Probability density functions for intensity induced phase noise in CW phase demodulation systems", Measurement Science and Technology, vol. 18, no. 11, pp. 3592.

[37] Roths, J. and Jülich, F. (2008), "Determination of strain sensitivity of free fiber Bragg gratings", Proc. SPIE, Vol. 7003, Optical Sensors 2008, pp. 700308.

[38] Frigo, N., Dandridge, A. and Tveten, A. (1984), "Technique for elimination of polarisation fading in fibre interferometers", Electronics Letters, vol. 20, no. 8, pp. 319-320.

[39] Lee, B. (2003), "Review of the present status of optical fiber sensors", Optical Fiber Technology, vol. 9, no. 2, pp. 57-79.

[40] James, S., Dockney, M. and Tatam, R. (1996), "Simultaneous independent temperature and strain measurement using in-fibre Bragg grating sensors", Electronics Letters, vol. 32, no. 12, pp. 1133-1134.

[41] Gao, S., O'Sullivan, M. and Hui, R. (2012), "Complex-optical-field lidar system for range and vector velocity measurement", Optics Express, vol. 20, no. 23, pp. 25867-25875. 